\title{
Konservativ behandeln oder operieren?
}

Fragestellung: Ist der Verschluss eines offenen Foramen ovale bei Patienten mit transienten ischämischen Attacken (TIAs) oder kryptogenem Schlaganfall einer medikamentösen Therapie überlegen?

Hintergrund: Seit mehr als 20 Jahren tobt eine erbitterte Schlacht in der Medizin über die Bedeutung des offenen Foramen ovale (PFO) bei kryptogenen Schlaganfällen. Ohne jeden Beweis der Wirksamkeit wurde bei Patienten mit kryptogenem Schlaganfall ein offenes Foramen ovale verschlossen unter der Vorstellung, damit erneute Schlaganfälle zu verhindern.

Patienten und Methodik: Es wurde eine Literaturrecherche aller Studien durchgeführt, bei denen Patienten mit PFO und kryptogenem Schlaganfall/TIA entweder einen Verschluss des PFO erhielten oder rein medikamentös mit Acetylsalicylsäure (ASS) oder Antikoagulanzien behandelt wurden. Keine der Studien war randomisiert. Der primäre Outcome der Analyse waren erneute Schlaganfälle und TIAs. Außerdem wurden die Komplikationen des PFO-Verschlusses erfasst ( $\bullet$ Abb.1).

Ergebnisse: Für die Analyse standen 39 Studien mit $8.185 \mathrm{~Pa}-$ tienten zur Verfügung, bei denen das PFO verschlossen wurde, und 19 Studien mit 2.142 konservativ behandelten Patienten. Die jährlichen Inzidenzraten nach PFO-Verschluss betru-

Agarwal S, Bajaj NS, Kumbhani DJ et al. Meta-analysis of transcatheter closure versus medical therapy for patent foramen ovale in prevention of recurrent neurological events after presumed paradoxical embolism. JACC Cardiovasc Intervent 2012; 5: $777-89$ legenheit des PFO-Verschlusses gegenüber der konservativen Therapie nahe. Ein Vergleich mit Plättchenhemmern zeigte eine Überlegenheit der Antikoagulation. 4\% der Patienten erlitten bei dem PFO-Verschluss eine ernsthafte Komplikation. Es bestand kein Zusammenhang zwischen der Rezidivrate und der Größe des Shunts. Besonders profitierten ältere Patienten mit einem Vorhofseptum-Aneurysma.

Schlussfolgerungen: Diese Übersicht kommt zu dem Schluss, dass der Verschluss eines offenen Foramen ovale beim kryptogenen Schlaganfall einer konservativen Therapie überlegen ist.

\section{-Kommentar von Prof. Hans-Christoph Diener \\ Missbrauch von Metaanalysen}

Dieser Artikel und die Metaanalyse sind ein gutes Beispiel für den Missbrauch von Literaturrecherchen und Metaanalysen. Als die Arbeit publiziert wurde, waren bereits die Ergebnisse der großen randomisierten CLOSURE-Studie bekannt, die keinerlei Nutzen des PFO-Verschlusses zur Rezidivprophylaxe bei Patienten mit kryptogenem Schlaganfall fand [1]. CLOSURE widerlegte auch alle Ergebnisse dieser Metaanalyse. Es ergab sich keine Überlegenheit der Antikoagulanzien gegenüber ASS und auch das Vorhofseptum-Aneurysma war kein Prädiktor für die Wirksamkeit des PFO-Verschlusses. Die wichtige Schlussfolgerung für die Leser: Metaanalysen sind beim Fehlen von sauber durchgeführten randomisierten Studien höchstens geeignet, um wissenschaftliche Hypothesen zu generieren. Therapieentscheidungen sollten sich aber ausschließlich auf die Ergebnisse großer randomisierten Studien stützen.

Referenz

1. Furlan AJ et al. N Engl J Med 2012; 366: $991-9$ 